\title{
Do-It-Yourself Transfer of Large-Area Graphene Using an Office Laminator and Water
}

Shivayogimath, Abhay; Whelan, Patrick Rebsdorf; MacKenzie, David M.A.; Luo, Birong; Huang, Deping; Luo, Da; Wang, Meihui; Gammelgaard, Lene; Shi, Haofei; Ruoff, Rodney S.

Total number of authors:

12

Published in:

Chemistry of Materials

Link to article, DOI:

10.1021/acs.chemmater.8b04196

Publication date:

2019

Document Version

Publisher's PDF, also known as Version of record

Link back to DTU Orbit

Citation (APA):

Shivayogimath, A., Whelan, P. R., MacKenzie, D. M. A., Luo, B., Huang, D., Luo, D., Wang, M., Gammelgaard, L., Shi, H., Ruoff, R. S., Bøggild, P., \& Booth, T. J. (2019). Do-It-Yourself Transfer of Large-Area Graphene Using an Office Laminator and Water. Chemistry of Materials, 31(7), 2328-2336.

https://doi.org/10.1021/acs.chemmater.8b04196

\section{General rights}

Copyright and moral rights for the publications made accessible in the public portal are retained by the authors and/or other copyright owners and it is a condition of accessing publications that users recognise and abide by the legal requirements associated with these rights.

- Users may download and print one copy of any publication from the public portal for the purpose of private study or research.

- You may not further distribute the material or use it for any profit-making activity or commercial gain

- You may freely distribute the URL identifying the publication in the public portal 


\title{
Do-It-Yourself Transfer of Large-Area Graphene Using an Office Laminator and Water
}

\author{
Abhay Shivayogimath, ${ }^{\dagger,+, \bigcirc \odot ~ P a t r i c k ~ R e b s d o r f ~ W h e l a n, ~}{ }^{\dagger, \ddagger, \S, \bigcirc \odot ~ D a v i d ~ M . A . ~ M a c k e n z i e, ~}$

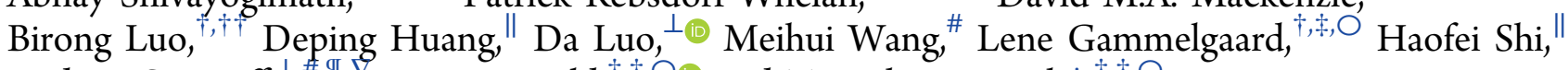 \\ Rodney S. Ruoff, ${ }^{\perp, \#, \Psi, \vartheta}$ Peter Bøggild, ${ }^{\dagger, \ddagger, \bigcirc}$ and Timothy J. Booth $*, \dagger, \ddagger, \bigcirc$ \\ ${ }^{\dagger}$ DTU Nanotech, Technical University of Denmark, Ørsteds Plads 345, DK-2800 Kgs. Lyngby, Denmark \\ ${ }^{\ddagger}$ Centre for Nanostructured Graphene (CNG), Technical University of Denmark, Ørsteds Plads 345C, DK-2800 Kgs. Lyngby, \\ Denmark \\ ${ }^{\S}$ DTU Fotonik, Technical University of Denmark, Ørsteds Plads 343, DK-2800 Kgs. Lyngby, Denmark \\ "Chongqing Institute of Green and Intelligent Technology, Chinese Academy of Sciences, 266 Fang Zheng Avenue, Chongqing \\ 400714, P. R. China \\ ${ }^{\perp}$ Center for Multidimensional Carbon Materials (CMCM), Institute for Basic Science (IBS), Ulsan 44919, Republic of Korea \\ \# Department of Chemistry, ${ }^{I}$ School of Materials Science and Engineering, and ${ }^{\nabla}$ School of Energy and Chemical Engineering, Ulsan \\ National Institute of Science and Technology (UNIST), Ulsan 44919, Republic of Korea
}

Supporting Information

\begin{abstract}
We demonstrate a simple method for transferring large areas (up to A4-size sheets) of CVD graphene from copper foils onto a target substrate using a commercially available polyvinyl alcohol polymer foil as a carrier substrate and commercial hot-roll office laminator. Through the use of terahertz time-domain spectroscopy and Raman spectroscopy, large-area quantitative optical contrast mapping, and the fabrication and electrical characterization of $\sim 50$ individual centimeter-scale van der Pauw field effect devices, we show a nondestructive technique to transfer large-area graphene with low residual doping that is scalable, economical, reproducible, and easy to use and that results in less doping and transferinduced damage than etching or electrochemical delamination

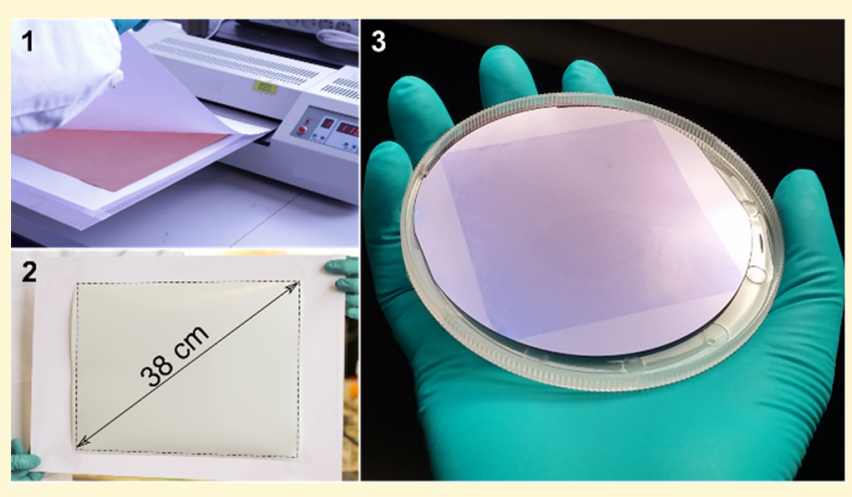
transfers. We show that the copper substrate can be used multiple times with minimal loss of material and no observable reduction in graphene quality. We have additionally demonstrated the transfer of multilayer hexagonal boron nitride from copper and iron foils. Finally, we note that this approach allows graphene to be supplied on stand-alone polymer supports by CVD graphene manufacturers to end users, with the only equipment and consumables required to transfer graphene onto target substrates being a commercial office laminator and water.
\end{abstract}

\section{INTRODUCTION}

Chemical vapor deposition (CVD) of graphene on commercial copper foils provides a scalable route toward producing continuous sheets of high-quality monolayer graphene that is being rapidly scaled toward industrial production. ${ }^{1}$ For most applications, graphene needs to be transferred from the growth substrate to a target substrate. This is typically done by applying a supporting polymer layer and then either chemically etching away the copper to release the graphene film $^{2-5}$ or electrochemically delaminating the graphene film from the copper substrate. ${ }^{6,7}$ Chemical etching provides a reliable method of transfer, but is relatively costly because the catalyst accounts for a significant portion of the graphene production costs and can result in undesirable residual contamination because of the wet etching process. ${ }^{1}$ Electrochemical delamination is a popular alternative which allows for reuse of the catalyst; however, this method has inherent throughput limitations because the transfer speed is limited by the area of the electrodes, which determines the areal rate at which the graphene is delaminated. The delamination speed must also be low to prevent excessive bubbling, which can damage the graphene film because of surface tension forces. ${ }^{8}$ Furthermore, although both methods have been shown to be scalable, a high level of skill and expertise is still needed to perform such transfers manually, which often translates into higher complexity and cost in terms of achieving a viable production scenario.

Received: October 2, 2018

Revised: March 1, 2019

Published: March 5, 2019 
This requirement also means that the transfer process must be carried out by the graphene supplier rather than the end-user, necessitating repeated shipment of an application-specific substrate to and from the graphene supplier. Therefore, it is advantageous for graphene manufacturers to supply CVD graphene on a generic support layer that allows easy, robust, and high-coverage transfer of the graphene onto target substrates by end-users without requiring a high level of expertise.

Recent years have shown progress in the development of convenient transfer processes with reduced contamination and the possibility of reusing the catalyst. ${ }^{9,10}$ These include methods where the underlying copper is oxidized in water, allowing the graphene to be subsequently peeled from the surface using a polymer support layer. ${ }^{10-12}$ This process takes place by intercalation of water in between the graphene and copper, after which the galvanic coupling between the more noble graphene film and copper surface leads to accelerated oxidation and corrosion of said surface $e^{13-15}$ and subsequent decoupling of graphene from the copper. This method is particularly attractive for scale-up because it uses only water and allows for parallelization of transfer by simultaneously oxidizing batches of graphene-coated catalyst in the water. While a number of support polymers such as polycarbonate and polymethyl methacrylate (PMMA) have been used for mechanically delaminating graphene from copper foils, ${ }^{12}$ these typically require removal in volatile toxic solvents such as chloroform and acetone, which necessitates special handling and disposal precautions. In this regard, polyvinyl alcohol (PVA) is an attractive alternative because it is water-soluble, allowing the entire transfer process to be done using safe and environmentally friendly solvents.

Here, we present a method for transferring large-area graphene using commercially available prefabricated PVA foils used for hydrographics printing, a commercial tabletop hot-roll office laminator, and water. This method provides a simple, convenient, and reproducible transfer technique that can easily be performed by end-users to transfer graphene onto a target substrate with high uniformity and coverage and lower residual doping than etching or electrochemical delamination transfers. We further show that the method is also effective for transferring other two-dimensional (2D) materials from metallic substrates, such as hexagonal boron nitride (hBN) from copper and iron foils.

\section{EXPERIMENTAL METHODS}

Graphene was synthesized on a $10 \mathrm{~cm} \times 10 \mathrm{~cm}$ commercial copper foil $(127 \mu \mathrm{m}$ thick, Alfa Aesar 13380) in a cold-wall CVD reactor (Black Magic, AIXTRON). The copper foil was electropolished in a phosphoric acid solution before CVD growth as described elsewhere. ${ }^{16}$ Graphene was fabricated as follows: the copper foil was annealed for $30 \mathrm{~min}$ at $1000{ }^{\circ} \mathrm{C}$ under $20 \mathrm{mbar}$ and $700 \mathrm{sccm}$ argon flow, followed by $60 \mathrm{~min}$ of $0.1 \mathrm{sccm} \mathrm{C_{4 }}, 50 \mathrm{sccm} \mathrm{H}$, and $100 \mathrm{sccm}$ $\mathrm{Ar}$ for graphene growth. The sample was subsequently cooled down to room temperature under $1000 \mathrm{sccm}$ argon flow.

Graphene for repeated growth and transfer experiments was synthesized on a $25 \mu \mathrm{m}$ thick electropolished commercial copper foil (Alfa Aesar, 13382). The mass of the foil was measured before and after each growth step using a microbalance and divided by the product of the area of the copper foil and the density of copper to obtain the average thickness lost during each growth and transfer step. The foil was cleaned in acetone and iso-propanol (IPA) and oxidized on a hot plate at $220{ }^{\circ} \mathrm{C}$ for $5 \mathrm{~min}$ prior to each growth step.

Large-area, monolayer graphene for $25 \mathrm{~cm} \times 30 \mathrm{~cm}$ transfers was grown on $25 \mu \mathrm{m}$ thick copper foils in a low pressure tube furnace system. The copper foil was annealed at $1000{ }^{\circ} \mathrm{C}$ with $500 \mathrm{sccm} \mathrm{H}_{2}$ and $700 \mathrm{sccm}$ argon flow, followed by $30 \mathrm{~min}$ of $100 \mathrm{sccm} \mathrm{CH}_{4}, 500$ $\mathrm{sccm} \mathrm{H}_{2}$, and $700 \mathrm{sccm}$ Ar for graphene growth. The sample was subsequently cooled down to room temperature under argon flow.

Monocrystalline graphene on $\mathrm{Cu}(111)$ was used for comparing etching, electrochemical delamination ("bubbling"), and PVA lamination transfers. Single-crystal $\mathrm{Cu}(111)$ foil was made from polycrystalline $\mathrm{Cu}$ foil (Nilaco CU-113253, $40 \mu \mathrm{m}$ thick, 99.9\%) by contact-free annealing. ${ }^{17}$ The single-crystal monolayer graphene film $(2 \mathrm{~cm} \times 3 \mathrm{~cm})$ was grown on this single-crystal $\mathrm{Cu}(111)$ foil in a low pressure tube furnace system. The $\mathrm{Cu}(111)$ foil was annealed to 1050 ${ }^{\circ} \mathrm{C}$ with $100 \mathrm{sccm} \mathrm{H}_{2}$ under 2.0 Torr in $1 \mathrm{~h}$, followed by $30 \mathrm{~min}$ of exposure to $1 \mathrm{sccm} \mathrm{CH}_{4}$ and $100 \mathrm{sccm} \mathrm{H}_{2}$ for graphene growth. The sample was then cooled to room temperature under 2.0 Torr with 100 sccm $\mathrm{H}_{2}$ by "sliding the furnace over." A detailed description of the single-crystal graphene film grown on the home-made $\mathrm{Cu}(111)$ foil will be published elsewhere.

Chemical etching and bubbling transfers of graphene on $\mathrm{Cu}(111)$ were performed as follows. For chemical etching transfer, a $4 \% \mathrm{wt}$ PMMA solution was spin-coated onto copper at $1500 \mathrm{rpm}$ for $1 \mathrm{~min}$ and baked at $80{ }^{\circ} \mathrm{C}$ for $5 \mathrm{~min}$. The foil was then floated on top of a $5 \% \mathrm{HCl} / \mathrm{H}_{2} \mathrm{O}_{2}$ etching solution overnight at room temperature to remove the copper. The PMMA/graphene film was then "fished out" and rinsed in deionized (DI) water, transferred onto a $90 \mathrm{~nm} \mathrm{SiO} 2 / \mathrm{Si}$ substrate, baked at $80{ }^{\circ} \mathrm{C}$ for $5 \mathrm{~min}$, and placed in acetone overnight to remove PMMA. For bubbling transfer, ${ }^{18}$ a PMMA layer was spincoated onto the copper at $3000 \mathrm{rpm}$ for $1 \mathrm{~min}$. The PMMA/ graphene $/ \mathrm{Cu}(111)$ foil stack was dipped into a $1 \mathrm{M} \mathrm{NaOH}$ aqueous solution to act as the cathode in an electrolysis cell run at constant current. The PMMA/graphene layer was delaminated from the $\mathrm{Cu}(111)$ foil after tens of seconds as a result of the formation of $\mathrm{H}_{2}$ bubbles at the interface between the graphene and the $\mathrm{Cu}(111)$ foil. ${ }^{18}$ After cleaning with DI water, the floating PMMA/graphene layer was "fished out" and transferred to the target substrate $(90 \mathrm{~nm} \mathrm{SiO} / 2 \mathrm{Si})$. Finally, the sample was dried and PMMA removed with acetone.

PVA lamination transfer of graphene was performed according to the procedure illustrated in Figure 1. Commercial water-soluble PVA films of $30 \mu \mathrm{m}$ thickness were purchased from Chengdu Han Shang Water Transfer Printing Co., Ltd (HS Inkjet Printable Blank PVA Water Transfer Printing Film). The films are transparent and come supported on a white sheet of paper. They were initially rinsed in IPA and dried under nitrogen flow. Copper foils with as-grown graphene

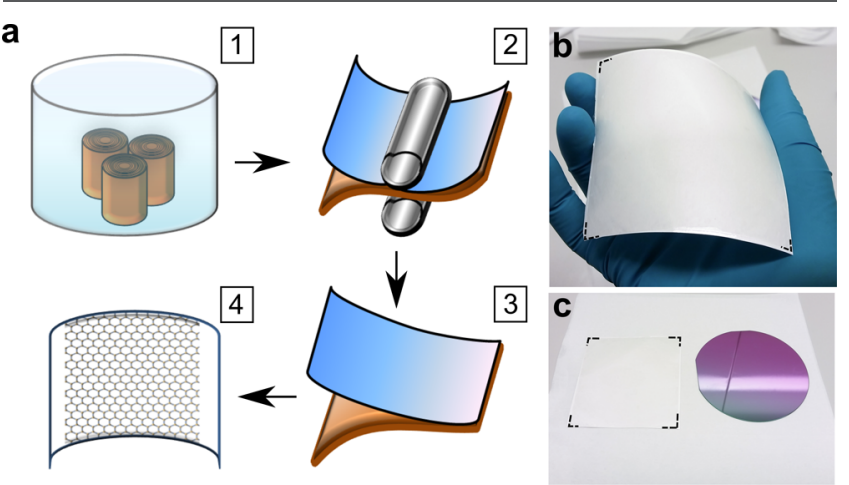

Figure 1. (a) Schematic illustration of the PVA lamination transfer process: (1) copper foils with as-grown graphene are oxidized in DI water at room temperature for more than $8 \mathrm{~h}$ (overnight); (2) the oxidized foils are subsequently laminated with a commercially available PVA film using a commercial hot-roll laminator. (3) After a $30 \mathrm{~s}$ bake on a hot plate at $110^{\circ} \mathrm{C}$, the PVA film is mechanically delaminated from the copper surface, taking the graphene film along with it (4). (b,c) Graphene transferred from a $10 \mathrm{~cm} \times 10 \mathrm{~cm}$ copper foil; the dashed lines serve as a visual guide marking the edges of the graphene sheet on the PVA film. (c) PVA-graphene film [from panel (b)] compared with a $4 " \mathrm{SiO}_{2}$ on $\mathrm{Si}$ wafer. 
were placed overnight in DI water at room temperature to oxidatively decouple the graphene from the catalyst surface. After drying the copper substrate, the IPA-rinsed PVA film was laminated on top of the graphene-copper foil at $110{ }^{\circ} \mathrm{C}$ and a speed of $20 \mathrm{~cm} / \mathrm{min}$ using a commercial hot-roll office laminator (Akiles Pro-Lam photo 6). The laminate was then baked on a hot plate at $110{ }^{\circ} \mathrm{C}$ for $30 \mathrm{~s}$ to improve adhesion between the PVA and graphene. The PVA film was then mechanically delaminated from the copper surface, lifting the graphene along with it. The graphene layer is now available as a transferrable layer on a mechanically stable and rigid polymer substrate, ready to be transported and eventually deposited on an arbitrary substrate. This was done by laminating the PVA-graphene film onto a target substrate under the same lamination conditions, baking it on a hot plate at $110{ }^{\circ} \mathrm{C}$ for $1 \mathrm{~min}$, peeling off the paper support while the sample is still on the hot plate and finally removing the PVA in room temperature DI water overnight.

Transferred graphene layers were characterized by Raman spectroscopy, optical microscopy, terahertz time-domain spectroscopy (THz-TDS), and four-point van der Pauw (vdP) electrical device measurements. Raman spectroscopy was conducted in a Thermo Fisher DXR microscope equipped with a $455 \mathrm{~nm}$ laser $(5 \mathrm{~mW}, 3 \times 10$ $\mathrm{s}$ exposure time, $50 \times$ objective). Raman spectral maps for comparative studies between etching, bubbling, and PVA lamination transfers were collected over 969 spectra spread evenly across a $150 \mu \mathrm{m} \times 72 \mu \mathrm{m}$ area. Optical images were acquired using a Nikon Eclipse L200N equipped with a programmable Prior Scientific XYZ stage. Optical images were stitched to form optical and graphene coverage maps of the samples. For the graphene coverage maps, each pixel in the optical images is filtered according to the expected red, green, and blue contrasts for graphene on a $90 \mathrm{~nm} \mathrm{SiO} 2 / \mathrm{Si}$ substrate. This makes it possible to produce a coverage map highlighting regions of singlelayer graphene, bilayer graphene, $\mathrm{SiO}_{2}$ substrate, and "other" - pixels here designated as "other" when they indicate more than two layers of graphene or with contaminants from the growth and transfer processes. ${ }^{19}$

THz-TDS measurements were conducted using a commercially available fiber-coupled spectrometer. ${ }^{20}$ Spatial THz-TDS maps were acquired by raster scanning the samples at normal incidence in the focal plane of the $\mathrm{THz}$ beam using $400 \mu \mathrm{m}$ step size for graphene on oxidized silicon and $1 \mathrm{~mm}$ step size for graphene on PVA. The THz spot size is $\sim 350 \mu \mathrm{m}$ at $1 \mathrm{THz}$. The frequency-dependent sheet conductivity of graphene was extracted from the ratio of $\mathrm{THz}$ radiation passing through graphene-covered sample regions and a substrate reference area with no graphene. ${ }^{20}$ The transient from the directly transmitted pulse was used for graphene on $90 \mathrm{~nm} \mathrm{SiO} 2$ on high resistivity (HR) Si wafer, ${ }^{21}$ whereas for graphene on PVA, all internally reflected echoes were taken into account as done in ref 22 . THz-TDS sheet conductivity maps show the spatially averaged sheet conductivity in the $0.3-1.1 \mathrm{THz}$ range. The DC conductivity $\sigma_{\mathrm{dc}}$ and scattering time $\tau$ can be extracted from THz-TDS measurements of graphene by fitting the frequency-dependent sheet conductivity of graphene to the Drude-model (Figure S1), $\sigma_{s}(\omega)=\sigma_{\mathrm{dc}} /(1-i \omega \tau)$, where $\sigma_{s}$ is the frequency-dependent sheet conductivity. ${ }^{21,23-25}$ The carrier density $n$ and mobility $\mu$ of graphene can subsequently be calculated as $n=\pi \hbar^{2} \sigma_{\mathrm{dc}}{ }^{2} /\left(e^{4} \nu_{\mathrm{F}}{ }^{2} \tau^{2}\right)$ and $\mu=\sigma_{\mathrm{dc}} /(e n),{ }^{21,23,25}$ where $\nu_{\mathrm{F}}$ is the Fermi velocity set to $10^{6} \mathrm{~m} / \mathrm{s}^{26}$

Devices for vdP measurements were fabricated according to the steps illustrated in Figure 2. $\mathrm{Cr} / \mathrm{Au}(5 / 45 \mathrm{~nm})$ metal electrodes were deposited through a shadow mask onto a $4^{\prime \prime}$ silicon wafer with $90 \mathrm{~nm}$ of thermally grown oxide on top ${ }^{27}$ (Figure 2a). An $80 \mathrm{~mm} \times 80 \mathrm{~mm}$ film of graphene was then transferred onto the wafer (Figure $2 b$ ) using the methods described above. The definition of device shape and graphene electrodes was carried out via laser ablation ${ }^{28}$ using a $1064 \mathrm{~nm}$ ps pulsed laser. The process yields 49 separate devices with individual device areas of $5 \mathrm{~mm} \times 5 \mathrm{~mm}$ (Figure 2c) in an hour of total processing time. ${ }^{29}$ Prior to measurement, devices were annealed at $225{ }^{\circ} \mathrm{C}$ in $\mathrm{N}_{2}$ for 30 min to remove surface adsorbents. ${ }^{30} \mathrm{VdP}$ measurements were performed by sequentially collecting electric field effect results for two four-point electrical configurations $R_{\mathrm{A}}$ and $R_{\mathrm{C}}$, (Figure $2 \mathrm{~d}$ ) with the sheet resistance $R_{\mathrm{S}}$ defined by: ${ }^{31}$
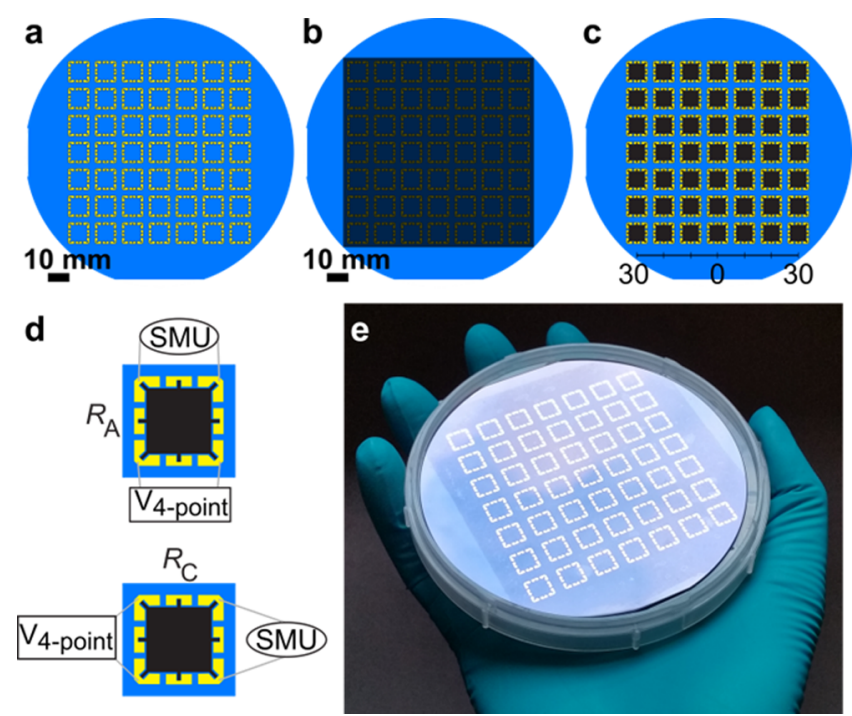

Figure 2. Overview of device processing steps and measurement configurations. (a) $\mathrm{Cr} / \mathrm{Au}$ electrodes are evaporated through a shadow mask onto a 4 " silicon wafer with $90 \mathrm{~nm}$ of oxide on top. (b) Graphene is transferred on top of the electrical contacts. (c) Devices are defined and electrically separated using laser ablation to remove unwanted graphene areas ${ }^{28}$ (scale: distance from device center in $\mathrm{mm}$ ). (d) Measurement configurations $A$ and $C$, used in the vdP measurements. (e) Photograph of 4" $90 \mathrm{~nm} \mathrm{SiO}_{2} / \mathrm{Si}$ wafer with graphene devices fabricated as described.

$$
e^{\pi R_{\mathrm{A}} / R_{\mathrm{S}}}+e^{\pi R_{\mathrm{C}} / R_{\mathrm{S}}}=1
$$

and with the gate-dependent device uniformity parameter defined as: ${ }^{32}$

$$
\beta=\frac{R_{\mathrm{A}}}{R_{\mathrm{C}}}
$$

\section{RESULTS}

Figure 3 shows optical and THz-TDS characterization of a graphene film transferred onto a $4^{\prime \prime} 90 \mathrm{~nm} \mathrm{SiO} 2$ on HR-Si wafer. The graphene transferred here was from the $100 \mathrm{~mm} \times$ $100 \mathrm{~mm}$ graphene on the PVA foil shown in Figure 1b. Optical microscopy and THz-TDS measurements both confirm that a continuous layer of electrically conductive graphene was transferred over the entire sample area. The THz-TDS map shows an average sheet conductivity of $\sim 0.5 \mathrm{mS}$ and is relatively homogeneous across the area. One can observe regions with darker contrast near the center and edges of the film in Figure $3 b, c$, which correspond to higher conductivity areas in the THz-TDS map (Figure $3 \mathrm{~d}$ ) - these are due to adlayers that originate from the growth process itself, as confirmed by scanning electron micrographs (SEM) of the catalyst surface before transfer (Figure S2). A region in the bottom right corner is visible in both the optical and THz-TDS maps where the graphene was damaged during handling.

For field effect measurements, graphene was transferred onto a $4^{\prime \prime} 90 \mathrm{~nm} \mathrm{SiO}_{2} / \mathrm{Si}$ wafer with 49 prefabricated device contacts (Figure 2a,b) and laser-patterned to form defined device geometries (Figure 2d). Back-gated electrical measurements were conducted on 10 different $5 \mathrm{~mm} \times 5 \mathrm{~mm}$ devices with varying distance to the wafer center (Figure 2c). Typical device characteristics are shown in Figure 4a. The charge neutrality point for all devices lies between -3 and $+1 \mathrm{~V}$, which corresponds to low residual doping levels below $8 \times 10^{11} \mathrm{~cm}^{-2}$. 

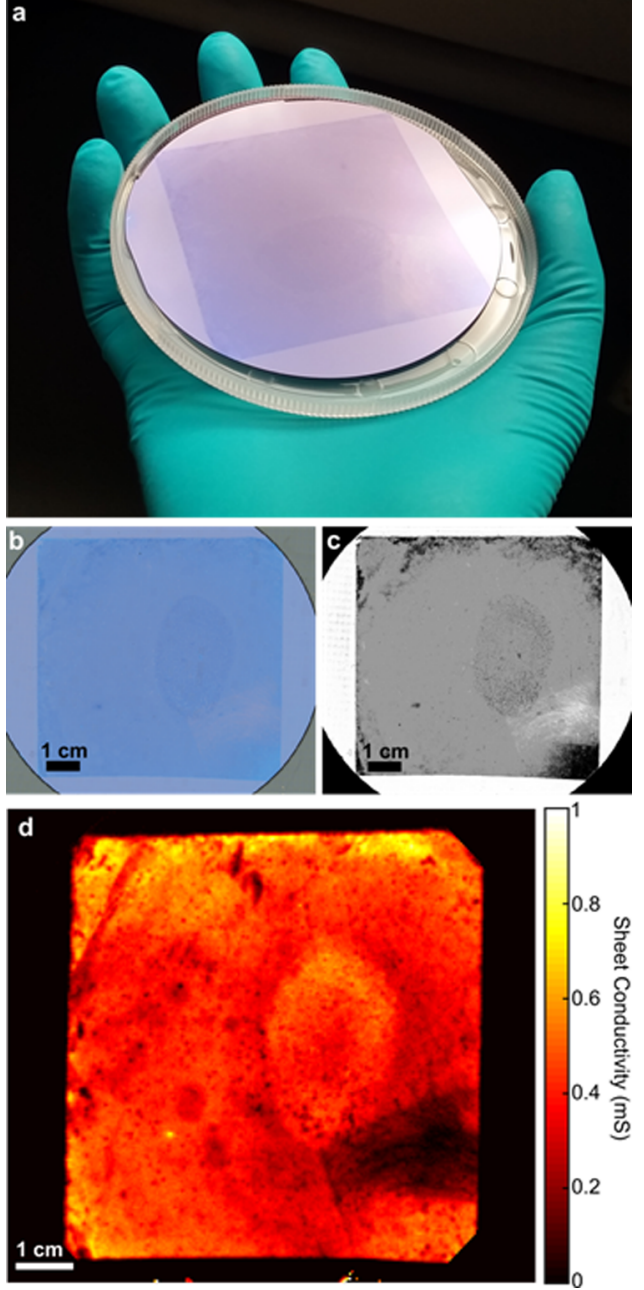

Figure 3. (a) Photograph of a 4" $90 \mathrm{~nm} \mathrm{SiO}{ }_{2}$ on HR-Si wafer with graphene transferred on top. (b) Stitched optical microscopy map of wafer from (a). (c) Coverage map from (b), where light gray represents single-layer graphene, dark gray represents the bilayer regions, white represents the substrate regions, and black represents the "other" regions: regions with more than two graphene layers or with contaminants from the growth and transfer processes. (d) $\mathrm{THz}-$ TDS sheet conductivity map of the graphene film transferred onto a $90 \mathrm{~nm} \mathrm{SiO} / \mathrm{Si}$ wafer. The average sheet conductivity in (c) is $\sim 0.5$ $\mathrm{mS}$.

The field-effect mobility was calculated via $\mu=(L /$ $W)\left(C_{\text {oxide }} V_{\mathrm{SD}}\right)^{-1}\left(\mathrm{~d} I / \mathrm{d} V_{\mathrm{G}}\right)^{33}$ and found to be in the range of $350-800 \mathrm{~cm}^{2} \mathrm{~V}^{-1} \cdot \mathrm{s}^{-1}$. We found the vdP uniformity parameter of the device to be $\beta<2$ (shown in Figure $4 \mathrm{a}$ inset), which is typical for high-quality large-scale CVD graphene devices that have been uniformly transferred within the device areas. ${ }^{32}$ When $n$ and $\mu$ are plotted as a function of position on the wafer, we do not observe any systematic variation between the wafer center and wafer edge, as shown in Figure $4 \mathrm{~b}$. All 49 devices were electrically conductive, indicating a $100 \%$ transfer yield.

PVA lamination transfer was benchmarked against standard chemical etching and bubbling transfer using Raman spectroscopy and THz-TDS measurements. Here, we used high-quality monocrystalline graphene on $\mathrm{Cu}(111)$ foils ${ }^{34,35}$ to mitigate the effects of defects from growth from those introduced during transfer. THz-TDS measurements were used as a noninvasive tool to extract charge carrier density and mobility that avoids
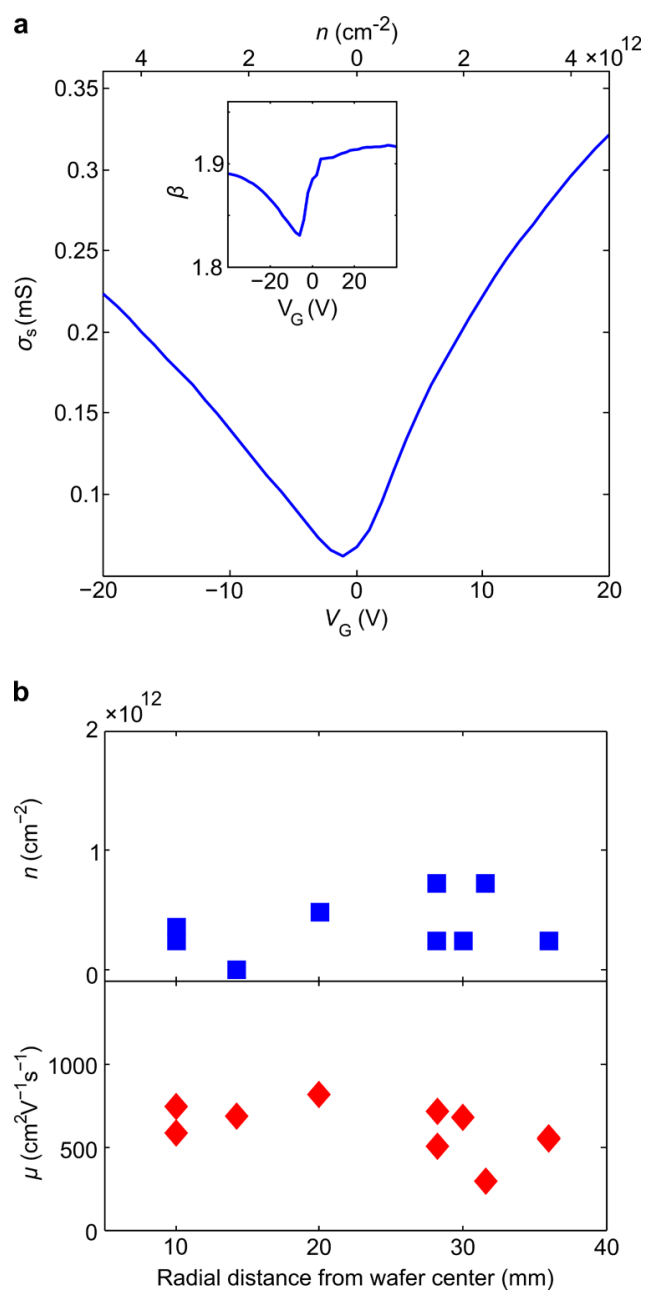

Figure 4. Electrical measurements from wafer-scale transfer. (a) Typical sheet conductivity $\left(\sigma_{\mathrm{s}}\right)$ measurements of a $5 \mathrm{~mm} \times 5 \mathrm{~mm}$ device. Inset: Device homogeneity $(\beta$ in eq 2$)$ as a function of gate bias. (b) Residual doping $(n)$ and carrier mobility $(\mu)$ as a function of the distance from wafer center (as defined in Figure $2 c$ ).

defects and doping induced by device fabrication. ${ }^{30}$ Extracted values for charge carrier density and mobility from THz-TDS measurements and Raman spectral figures of merit are shown in Figure 5. Graphene from PVA lamination transfer exhibits lower charge carrier densities than either etching or bubbling transfers (Figure 5a), which corroborates the low residual doping seen from gated conductance measurements in Figure 4. This method also results in the lowest distribution in carrier density, which suggests that what little doping is introduced by transfer is more homogeneously distributed. Extracted mobility values are also $\sim 2 \times$ higher for PVA lamination transfer as compared to etching or bubbling transfers (Figure 5b). Raman spectra of graphene from PVA lamination transfer have higher average $2 \mathrm{D} / \mathrm{G}$ (Figure $5 \mathrm{c}$ ) and lower $\mathrm{D} / \mathrm{G}$ ratios (Figure $5 \mathrm{~d}$ ) and a lower 2D full-width at half maximum (FWHM) (Figure 5e) compared with the other two methods. In general, we observe bubbling transfer to result in higher doping, lower carrier mobility, and higher incidence of defects (as inferred from Figure 5d and Raman D-peak intensity in Figure S3) than the other two transfers. The results suggest that after controlling for growth- or fabrication-induced defects and doping, etching or bubbling transfer have a greater detrimental 

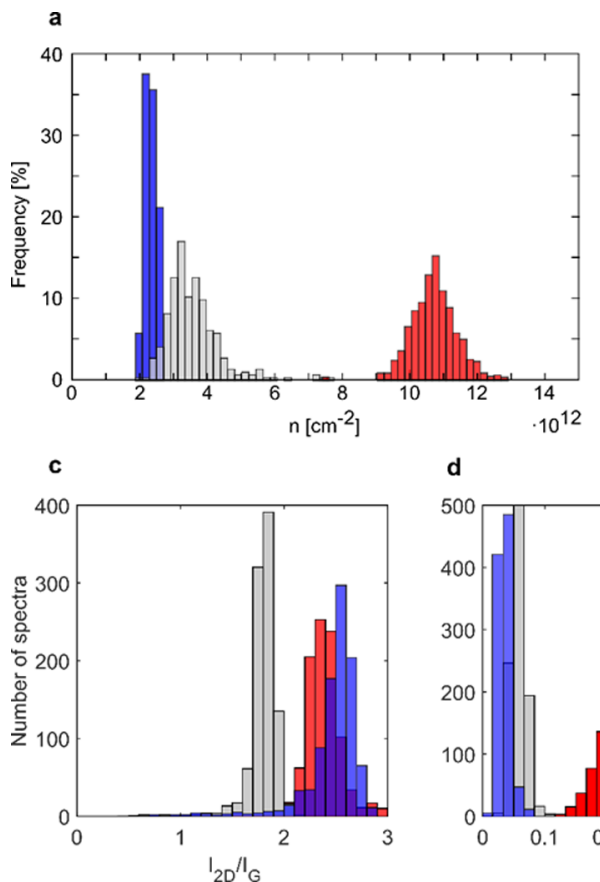

d
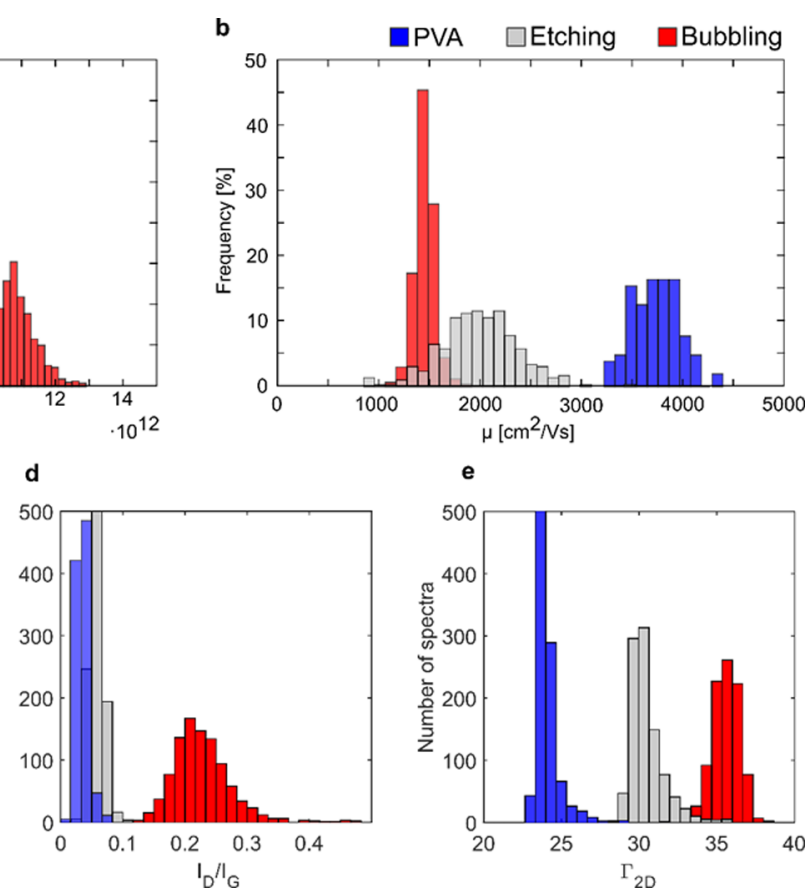

e

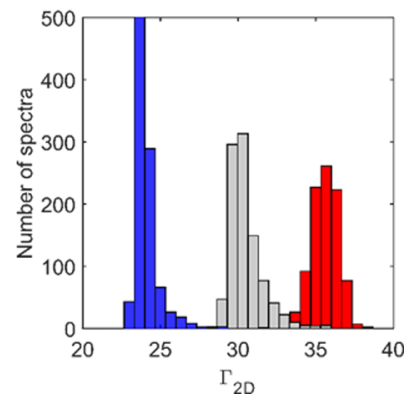

Figure 5. THz-TDS and Raman characterization of monocrystalline graphene on $\mathrm{Cu}(111)$ transferred by PVA lamination transfer (blue), etching transfer (gray), and bubbling transfer (red). (a,b) Histograms of carrier density (a) and mobility (b) from THz-TDS measurements. Average values of Gaussian fits to the histograms are (a) $2.42(14) \times 10^{12} \mathrm{~cm}^{-2}$ (PVA), $3.58(78) \times 10^{12} \mathrm{~cm}^{-2}$ (etching), $1.07(7) \times 10^{13} \mathrm{~cm}^{-2}$ (bubbling), (b) $3707(310) \mathrm{cm}^{2} / \mathrm{V} \mathrm{s}$ (PVA), 1983(386) $\mathrm{cm}^{2} / \mathrm{V} \mathrm{s}$ (etching), 1443(98) $\mathrm{cm}^{2} / \mathrm{V} \mathrm{s}$ (bubbling). Raman (c) 2D/G and (d) D/G intensity ratios and (e) FWHM of the 2D peak.

impact on graphene properties as compared to our PVA lamination transfer.

To demonstrate the scalability of our transfer method, CVD graphene grown on a $30 \mathrm{~cm} \times 25 \mathrm{~cm}$ electropolished copper foil was transferred onto a PVA support film, as shown in Figure 6. The graphene film is visible by eye as a continuous region of darker contrast on the PVA foil (Figure 6a). THzTDS mapping of the average sheet conductivity of graphene ${ }^{23}$ on the PVA foil shows a continuous conductive layer transferred onto the PVA foil with an average sheet conductivity of $\sim 1 \mathrm{mS}$. Streaking lines observed in the THzTDS conductivity map (Figure $6 \mathrm{~b}$ ) are areas where the graphene was not properly transferred because the PVA foil was not in close contact with the copper, which we attribute to thermal and mechanical stresses in the PVA foil during the lamination process.

Figure 7 shows results from repeated growth and transfer of graphene from a single $25 \mu \mathrm{m}$ electropolished copper foil as characterized by optical and Raman spectroscopy. Continuous sheets of graphene were transferred in all five trials, as seen in the optical micrographs in Figure $7 \mathrm{a}-\mathrm{e}$. Tears, holes, and polymer residues are occasionally observed in the optical images. Raman characterization in Figure $7 \mathrm{f}$ corroborates these observations by the presence of a small D-peak located at 1350 $\mathrm{cm}^{-1}$; the frequency and magnitude of these defects, however, appear to be random across repeated trials and thus not inherent to the transfer process itself. We found that a large number of creases formed on the thin $25 \mu \mathrm{m}$ foils when mechanically delaminating the PVA film, which persisted and adversely affected the quality of subsequent growth and transfers, leading to many of the macroscopic tears seen in transferred samples. Using a thicker foil (such as in Figure 3) virtually eliminated these creases.

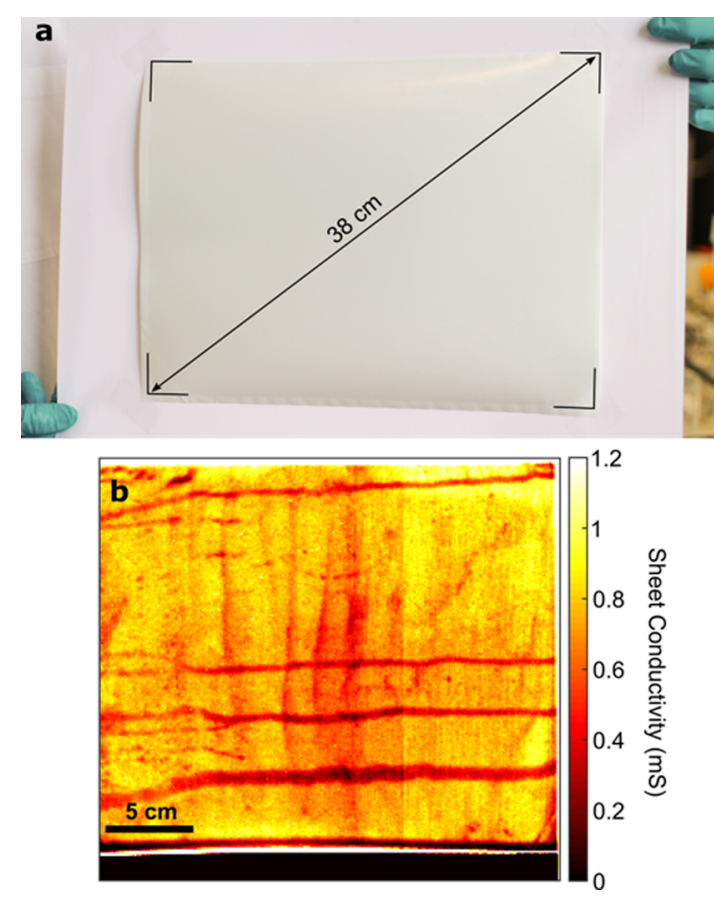

Figure 6. (a) Photograph of a $25 \times 30 \mathrm{~cm}^{2}$ sheet of monolayer graphene transferred onto a PVA support film, with the dashed lines denoting the edges of the graphene film. (b) THz-TDS sheet conductivity map of the graphene on PVA foil in (a), showing an average sheet conductivity of approximately $1 \mathrm{mS}$.

The amount of copper lost following each growth and transfer cycle was recorded by measuring the mass of the foil on a microbalance before and after each growth, and is presented in Figure 7g. From these measurements, we observe 

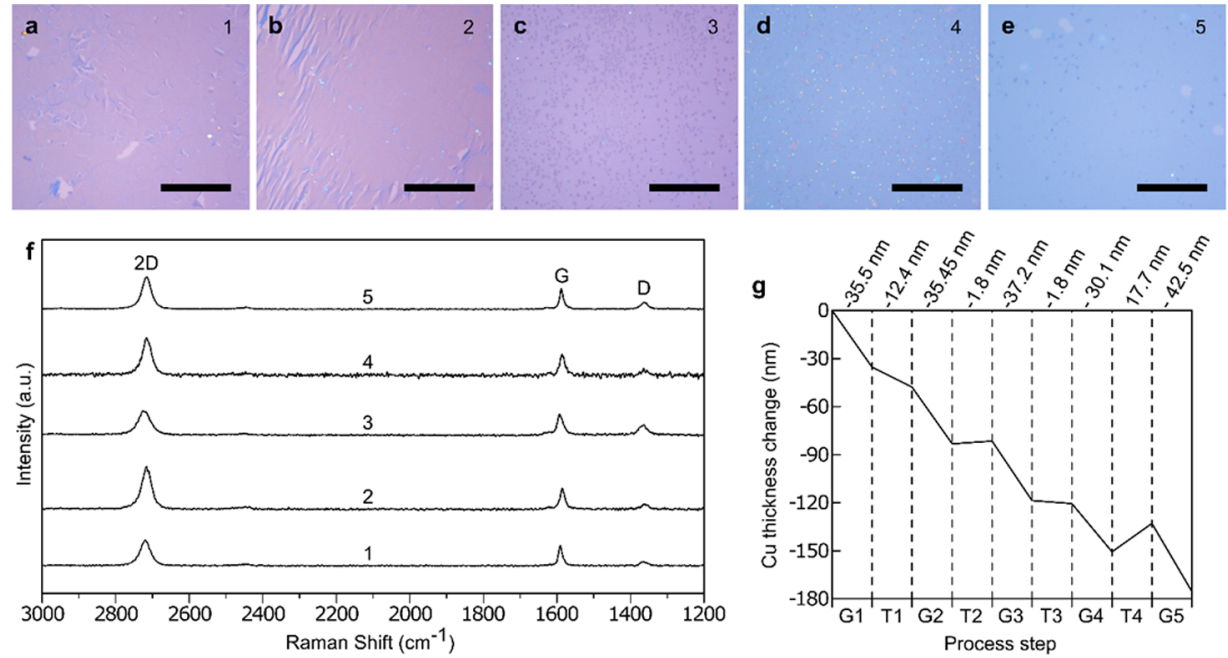

Figure 7. (a-e) Optical micrographs of repeated growth and transfer of graphene onto $90 \mathrm{~nm} \mathrm{SiO}_{2} / \mathrm{Si}$ substrates, where the sequential transfer index is indicated in the top right corner (scale bar $20 \mu \mathrm{m}$ ). (f) Corresponding Raman spectra of the samples in (a-e). (g) Plot of the average thickness of copper lost during each process step, where "G" refers to the growth step and " $T$ " refers to the transfer step; the numbers at the top axis indicate the average thickness of copper lost during each step, where a negative sign indicates a net loss in mass and a positive value indicates a gain in mass. The mass here was measured on a microbalance before and after each growth step. The instrumental precision of the microbalance, when converted to corresponding units of thickness, is $\pm 1.7 \mathrm{~nm}$.

that copper loss from the transfer process is minimal (within an instrumental error of $\pm 1.7 \mathrm{~nm}$ ) and that most of the loss occurs via evaporation during low pressure growth. Figure $7 \mathrm{~g}$ also shows that the copper lost during the first transfer (T1) was more significant than in subsequent transfers-the copper foil was oxidized in hot water after the first growth, forming black cuprous oxides, which subsequently delaminated along with the graphene during transfer.

The present process can also be used to transfer other $2 \mathrm{D}$ materials from metal surfaces. Figure 8 shows the results of transferring multilayer boron nitride sheets from copper and iron foils onto $90 \mathrm{~nm} \mathrm{SiO}_{2} / \mathrm{Si}$ substrates. Multilayer boron nitride was grown using methods described in literature. ${ }^{36,37}$ Of particular note is the fact that the copper foils did not need to be oxidized to peel off the boron nitride film. Water oxidation led to more wrinkles in transferred hBN films

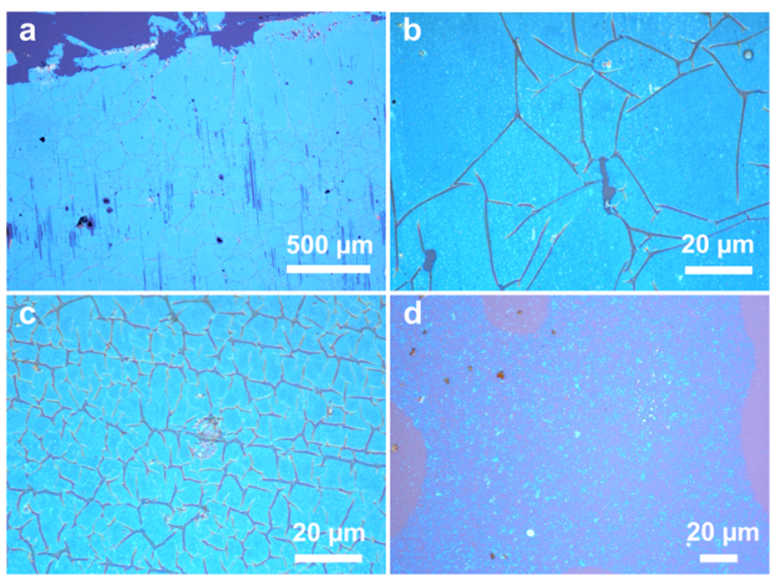

Figure 8. (a,b) Multilayer boron nitride (30 nm thick) transferred onto $90 \mathrm{~nm} \mathrm{SiO} 2 / \mathrm{Si}$ substrates from copper foils without a prior wet oxidation step. (c) Multilayer boron nitride transferred in the same manner as (a,b), but with a prior wet oxidation step. (c) Multilayer boron nitride transferred from an iron foil onto $90 \mathrm{~nm} \mathrm{SiO} / 2 / \mathrm{Si}$ substrate without prior wet oxidation.
(Figure 8c) than when oxidation was omitted (Figure 8b). Multilayer boron nitride films grown on iron foils ${ }^{37}$ had a nonuniform thickness. The films were transferred onto $90 \mathrm{~nm}$ $\mathrm{SiO}_{2} / \mathrm{Si}$ substrates without oxidizing the foils in water; the transferred films were discontinuous with primarily multilayer regions being transferred (Figure $8 \mathrm{~d}$ ). Water oxidation is not suitable for iron foils because of excessive rust formation, which is then transferred along with the films in subsequent steps.

\section{DISCUSSION}

Our results demonstrate that PVA lamination transfer is a commercially viable approach for transferring large-scale graphene from the copper catalyst onto target substrates in a convenient and reproducible manner. We show from $\mathrm{THz}-$ TDS and Raman spectroscopy that graphene transferred via PVA lamination has lower and more homogeneous residual doping, higher charge carrier mobility, and fewer transferinduced defects as compared to standard chemical etching or electrochemical delamination transfers. Through fabrication and characterization of large-scale electrical devices, we show that PVA lamination transfer provides high device yields with wafer-scale transport consistency. We further demonstrate, through the use of large-area THz-TDS characterization, that electrically continuous graphene films can be easily transferred over arbitrary length scales in a roll-to-roll fashion that is limited only by the dimensions of the catalyst. Finally, we show that other 2D materials can also be transferred from various substrates by this approach, as long as the material can be sufficiently decoupled from the substrate.

While PVA lamination transfer provides a high yield of devices with minimal residual doping, we nonetheless observe a small asymmetry in the conductivity of holes and electrons and a slight $\mathrm{n}$-doping in gated conductance measurements in Figure $4 \mathrm{a}$, which we attribute to PVA residues. ${ }^{38} \mathrm{We}$ also note that the carrier mobility values in these large-scale devices are lower than those reported in the literature for other transfer methods for micrometer-scale devices; ${ }^{6,7,9,39}$ the devices 
presented here have an area 5 orders of magnitude larger than a typical $10 \mu \mathrm{m} \times 10 \mu \mathrm{m}$ sized device. As the device area increases, large-scale inhomogeneities from growth, transfer, wafer variation, inconsistent polymer residues and so forth, which are less pronounced in individual small-scale devices, can play a significant role and lead to a lower measured mobility. ${ }^{32,40}$ Furthermore, doping and damage induced during growth and/or device fabrication ${ }^{30}$ can often mask or exacerbate the effects of transfer, making comparisons between different transfer methods difficult without accounting for these effects.

For more accurate comparisons between transfer methods, we used high-quality monocrystalline graphene on $\mathrm{Cu}(111)$ foils to decouple defects introduced during growth from transfer and THz-TDS and Raman measurements as noninvasive, noncontact tools to characterize the electrical and structural quality of the graphene immediately after transfer and prior to any processing. In doing so, we were able to see clear differences in the graphene quality obtained between the three transfer methods.

Transfer of multilayer hBN showed some important differences from graphene transfer. In particular, water oxidation was not necessary for transfer and in fact led to more wrinkles in the $\mathrm{hBN}$ film. A possible mechanism for this wrinkling is weak binding with the growth substrate after water oxidation, combined with different rates of thermal expansion during hot lamination. The wrinkles are also large because of a lower ability of thicker hBN to conform to the target substrate as compared to monolayer graphene. Direct delamination of $\mathrm{hBN}$ was also possible from iron foils; however, the transferred films were discontinuous. This may be a product of the nonuniform thickness of the grown film and stronger adhesion of boron nitride to iron as compared with copper, ${ }^{41}$ where the transfer mechanism at work resembles mechanical exfoliation of bulk h-BN rather than oxidative decoupling.

In general, we find that the quality and surface roughness of as-grown graphene on copper and contact between the PVA foil and substrate surface play an important role in the amount of defects (such as tears and holes) that is introduced during transfer. Microscopic tears appear to originate from poorly stitched graphene grain boundaries or tears and wrinkles already present in the as-grown graphene on copper. Macroscopic tears are seen in areas where the PVA foil is unable to make a close physical contact with the surface, such as rolling lines, creases, or mechanical deformations in the catalyst foil. Finally, holes are typically seen in regions with trapped air bubbles from lamination.

We envision a number of routes for increasing the throughput and quality of the present method for industrial scale-up. Although long processing times are used here to ensure complete decoupling of graphene from the catalyst surface during water oxidation and removal of PVA, hot water can oxidize copper and decouple graphene in less than an hour, ${ }^{11}$ and thinner PVA foils can be used to permit faster dissolution. Throughput can be increased further by performing these steps in large batches. Finally, roll-to-roll processing under tension, vacuum lamination, and thick $\mathrm{Cu}$ foils $(>50$ $\mu \mathrm{m})$ can be employed to reduce trapped bubbles, tearing, and other possible issues when scaling up this technology.

PVA lamination transfer provides a unique solution for industrial-scale graphene transfer: prefabricated PVA foils are inexpensive and readily available in industrial quantities and provide a mechanically robust support on which graphene can be supplied, while the simplicity of this lamination technique lowers the entry barrier for graphene transfer onto arbitrary substrates. As a result, instead of having to develop the expertise and provide graphene already transferred to application-specific substrates, graphene manufacturers can now provide high-quality CVD graphene on standalone, readyto-transfer foils that can be cut, shaped, and transferred as needed onto application-specific target substrates by the endusers using off-the-shelf office tools and water. Not only does this reduce production costs on the side of the manufacturer by allowing reuse of the copper catalyst, but it also makes CVD graphene available to a much larger base of potential usersnot just the experts - and enables do-it-yourself application of graphene films onto various target substrates by end-users with minimal investment of time and money.

\section{CONCLUSION}

We have demonstrated how commercially available PVA films widely used for the hydrographic transfer of printed images can be employed to mechanically transfer graphene from growth substrates to target substrates using a low-cost office laminator. The PVA foils act not only as a robust backing during handling but are also a convenient means to store or transport ready-toapply graphene to end-users, without previous experience with graphene transfer, who simply need to use their own low-cost laminator to apply the graphene on-site to any substrate of interest. By avoiding resist spinning or other complex processing, the need for catalyst etching and recovery and the use of organic solvents; and using only nontoxic and biodegradable materials, we have demonstrated a process which is greener, of lower cost, highly scalable, and more convenient for graphene growers and end-users than existing techniques and has considerable potential for further development and use in research and industry.

\section{ASSOCIATED CONTENT}

S Supporting Information

The Supporting Information is available free of charge on the ACS Publications website at DOI: 10.1021/acs.chemmater.8b04196.

Frequency-dependent conductivity from THz-TDS measurements of transferred graphene, optical and SEM characterization of graphene from Figure 3, and characteristic Raman spectrum of graphene transferred by PVA lamination, bubbling, and etching transfers (PDF)

\section{AUTHOR INFORMATION}

\section{Corresponding Author}

*E-mail: tibo@dtu.dk.

ORCID

Abhay Shivayogimath: 0000-0002-5152-0327

Patrick Rebsdorf Whelan: 0000-0002-3978-7029

David M.A. Mackenzie: 0000-0003-1114-2955

Da Luo: 0000-0002-9128-6782

Peter Bøggild: 0000-0002-4342-0449

Present Addresses

ODTU Physics, Technical University of Denmark, Ørsteds Plads 345, DK-2800 Kgs. Lyngby, Denmark

Department of Electronics and Nanoengineering, Aalto University, P.O. Box 13500, FI-00076 Aalto, Finland 
${ }^{\dagger \dagger}$ College of Physics and Materials Science, Tianjin Normal University 300387 Tianjin, P.R. China.

\section{Author Contributions}

A.S. conceived and developed the transfer process under the supervision of T.J.B. and P.B. P.R.W performed optical contrast mapping and THz-TDS characterization. D.M.A.M. performed device fabrication and electrical characterization. L.G. aided in device fabrication and characterization. B.L. provided samples of CVD h-BN grown on copper. H.D. and H.S. provided A4-size sheets of as-grown graphene on copper. D.L. and R.S.R provided graphene on $\mathrm{Cu}$ (111), and M.W. performed electrochemical delamination transfer. T.J.B. and P.B. supervised the project. A.S. wrote the manuscript and prepared the figures, and all authors contributed to manuscript revisions.

Notes

The authors declare no competing financial interest.

\section{ACKNOWLEDGMENTS}

A.S., B.L., P.B., and T.J.B. acknowledge the support from the EU Seventh Framework Programme (FP7/2007-2013) under grant agreement number FP7-6040007 “GLADIATOR”. P.R.W., D.M.A.M., L.G., and P.B. acknowledge the support from the EU Horizon 2020 Future and Emerging Technologies programme under grant agreement numbers 696656 and 785219. D.L. and R.S.R. acknowledge the support from IBSR019-D1. A.S., T.J.B., and P.B. acknowledge the support from the Danish National Research Foundation Center of Excellence for Nanostructured Graphene (CNG), project DNRF103.

\section{REFERENCES}

(1) Zhu, Y.; Ji, H.; Cheng, H.-M.; Ruoff, R. S. Mass Production and Industrial Applications of Graphene Materials. Natl. Sci. Rev. 2017, 5, 90-101.

(2) Bae, S.; Kim, H.; Lee, Y.; Xu, X.; Park, J.-S.; Zheng, Y.; Balakrishnan, J.; Lei, T.; Ri Kim, H.; Song, Y. I.; et al. Roll-to-Roll Production of 30-Inch Graphene Films for Transparent Electrodes. Nat. Nanotechnol. 2010, 5, 574-578.

(3) Li, X.; Zhu, Y.; Cai, W.; Borysiak, M.; Han, B.; Chen, D.; Piner, R. D.; Colombo, L.; Ruoff, R. S. Transfer of Large-Area Graphene Films for High-Performance Transparent Conductive Electrodes. Nano Lett. 2009, 9, 4359-4363.

(4) Lee, Y.; Bae, S.; Jang, H.; Jang, S.; Zhu, S.-E.; Sim, S. H.; Song, Y. I.; Hong, B. H.; Ahn, J.-H. Wafer-Scale Synthesis and Transfer of Graphene Films. Nano Lett. 2010, 10, 490-493.

(5) Chen, X.-D.; Liu, Z.-B.; Zheng, C.-Y.; Xing, F.; Yan, X.-Q.; Chen, Y.; Tian, J.-G. High-Quality and Efficient Transfer of Large-Area Graphene Films onto Different Substrates. Carbon 2013, 56, 271278.

(6) Wang, Y.; Zheng, Y.; Xu, X.; Dubuisson, E.; Bao, Q.; Lu, J.; Loh, K. P. Electrochemical Delamination of CVD-Grown Graphene Film: Toward the Recyclable Use of Copper Catalyst. ACS Nano 2011, 5, 9927-9933.

(7) Gao, L.; Ren, W.; Xu, H.; Jin, L.; Wang, Z.; Ma, T.; Ma, L.-P.; Zhang, Z.; Fu, Q.; Peng, L.-M.; et al. Repeated Growth and Bubbling Transfer of Graphene with Millimetre-Size Single-Crystal Grains Using Platinum. Nat. Commun. 2012, 3, 699.

(8) de la Rosa, C. J. L.; Lindvall, N.; Cole, M. T.; Nam, Y.; Löffler, M.; Olsson, E.; Yurgens, A. Frame Assisted $\mathrm{H}_{2} \mathrm{O}$ Electrolysis Induced $\mathrm{H}_{2}$ Bubbling Transfer of Large Area Graphene Grown by Chemical Vapor Deposition on Cu. Appl. Phys. Lett. 2013, 102, 022101.

(9) Wang, R.; Whelan, P. R.; Braeuninger-Weimer, P.; Tappertzhofen, S.; Alexander-Webber, J. A.; Van Veldhoven, Z. A.; Kidambi, P. R.; Jessen, B. S.; Booth, T.; Bøggild, P.; et al. Catalyst
Interface Engineering for Improved 2D Film Lift-off and Transfer. ACS Appl. Mater. Interfaces 2016, 8, 33072-33082.

(10) Yang, S. Y.; Oh, J. G.; Jung, D. Y.; Choi, H.; Yu, C. H.; Shin, J.; Choi, C.-G.; Cho, B. J.; Choi, S.-Y. Metal-Etching-Free Direct Delamination and Transfer of Single-Layer Graphene with a High Degree of Freedom. Small 2015, 11, 175-181.

(11) Whelan, P. R.; Jessen, B. S.; Wang, R.; Luo, B.; Stoot, A. C.; Mackenzie, D. M. A.; Braeuninger-Weimer, P.; Jouvray, A.; Prager, L.; Camilli, L.; et al. Raman Spectral Indicators of Catalyst Decoupling for Transfer of CVD Grown 2D Materials. Carbon 2017, 117, 75-81.

(12) Luo, D.; You, X.; Li, B.-W.; Chen, X.; Park, H. J.; Jung, M.; Ko, T. Y.; Wong, K.; Yousaf, M.; Chen, X.; et al. Role of Graphene in Water-Assisted Oxidation of Copper in Relation to Dry Transfer of Graphene. Chem. Mater. 2017, 29, 4546-4556.

(13) Schriver, M.; Regan, W.; Gannett, W. J.; Zaniewski, A. M.; Crommie, M. F.; Zettl, A. Graphene as a Long-Term Metal Oxidation Barrier: Worse Than Nothing. ACS Nano 2013, 7, 5763-5768.

(14) Wu, R.; Gan, L.; Ou, X.; Zhang, Q.; Luo, Z. Detaching Graphene from Copper Substrate by Oxidation-Assisted Water Intercalation. Carbon 2016, 98, 138-143.

(15) Wong, K.; Kang, S. J.; Bielawski, C. W.; Ruoff, R. S.; Kwak, S. K. First-Principles Study of the Role of $\mathrm{O}_{2}$ and $\mathrm{H}_{2} \mathrm{O}$ in the Decoupling of Graphene on $\mathrm{Cu}(111)$. J. Am. Chem. Soc. 2016, 138, 10986-10994.

(16) Miseikis, V.; Convertino, D.; Mishra, N.; Gemmi, M.; Mashoff, T.; Heun, S.; Haghighian, N.; Bisio, F.; Canepa, M.; Piazza, V.; et al. Rapid CVD Growth of Millimetre-Sized Single Crystal Graphene Using a Cold-Wall Reactor. 2D Mater. 2015, 2, 014006.

(17) Jin, S.; Huang, M.; Kwon, Y.; Zhang, L.; Li, B.-W.; Oh, S.; Dong, J.; Luo, D.; Biswal, M.; Cunning, B. V.; et al. Colossal Grain Growth Yields Single-Crystal Metal Foils by Contact-Free Annealing. Science 2018, 362, 1021-1025.

(18) Huang, M.; Biswal, M.; Park, H. J.; Jin, S.; Qu, D.; Hong, S.; Zhu, Z.; Qiu, L.; Luo, D.; Liu, X.; et al. Highly Oriented Monolayer Graphene Grown on a $\mathrm{Cu} / \mathrm{Ni}(111)$ Alloy Foil. ACS Nano 2018, 12, $6117-6127$

(19) Jessen, B. S.; Whelan, P. R.; Mackenzie, D. M. A.; Luo, B.; Thomsen, J. D.; Gammelgaard, L.; Booth, T. J.; Bøggild, P. Quantitative Optical Mapping of Two-Dimensional Materials. Sci. Rep. 2018, 8, 6381.

(20) Buron, J. D.; Petersen, D. H.; Bøggild, P.; Cooke, D. G.; Hilke, M.; Sun, J.; Whiteway, E.; Nielsen, P. F.; Hansen, O.; Yurgens, A.; et al. Graphene Conductance Uniformity Mapping. Nano Lett. 2012, 12, 5074-5081.

(21) Buron, J. D.; Mackenzie, D. M. A.; Petersen, D. H.; Pesquera, A.; Centeno, A.; Bøggild, P.; Zurutuza, A.; Jepsen, P. U. Terahertz Wafer-Scale Mobility Mapping of Graphene on Insulating Substrates without a Gate. Opt. Express 2015, 23, 30721.

(22) Whelan, P. R.; Huang, D.; Mackenzie, D.; Messina, S. A.; Li, Z.; Li, X.; Li, Y.; Booth, T. J.; Jepsen, P. U.; Shi, H.; et al. Conductivity Mapping of Graphene on Polymeric Films by Terahertz TimeDomain Spectroscopy. Opt. Express 2018, 26, 17748.

(23) Bøggild, P.; Mackenzie, D. M. A.; Whelan, P. R.; Petersen, D. H.; Buron, J. D.; Zurutuza, A.; Gallop, J.; Hao, L.; Jepsen, P. U. Mapping the Electrical Properties of Large-Area Graphene. 2D Mater. 2017, 4, 042003.

(24) Buron, J. D.; Pizzocchero, F.; Jessen, B. S.; Booth, T. J.; Nielsen, P. F.; Hansen, O.; Hilke, M.; Whiteway, E.; Jepsen, P. U.; Bøggild, P.; et al. Electrically Continuous Graphene from Single Crystal Copper Verified by Terahertz Conductance Spectroscopy and Micro FourPoint Probe. Nano Lett. 2014, 14, 6348-6355.

(25) Whelan, P. R.; Iwaszczuk, K.; Wang, R.; Hofmann, S.; Bøggild, P.; Jepsen, P. U. Robust Mapping of Electrical Properties of Graphene from Terahertz Time-Domain Spectroscopy with Timing Jitter Correction. Opt. Express 2017, 25, 2725-2732.

(26) Neto, A. H. C.; Guinea, F.; Peres, N. M. R.; Novoselov, K. S.; Geim, A. K. The Electronic Properties of Graphene. Rev. Mod. Phys. 2009, 81, 109-162. 
(27) Mackenzie, D. M. A.; Smistrup, K.; Whelan, P. R.; Luo, B.; Shivayogimath, A.; Nielsen, T.; Petersen, D. H.; Messina, S. A.; Bøggild, P. Batch Fabrication of Nanopatterned Graphene Devices via Nanoimprint Lithography. Appl. Phys. Lett. 2017, 111, 193103.

(28) Mackenzie, D. M. A.; Buron, J. D.; Whelan, P. R.; Jessen, B. S.; Silajdźić, A.; Pesquera, A.; Centeno, A.; Zurutuza, A.; Bøggild, P.; Petersen, D. H. Fabrication of CVD Graphene-Based Devices via Laser Ablation for Wafer-Scale Characterization. 2D Mater. 2015, 2, 045003.

(29) Mackenzie, D. M. A.; Buron, J. D.; Boaggild, P.; Jepsen, P. U.; Petersen, D. H. Contactless Graphene Conductance Measurements: The Effect of Device Fabrication on Terahertz Time-Domain Spectroscopy. Int. J. Nanotechnol. 2016, 13, 591.

(30) Gammelgaard, L.; Caridad, J. M.; Cagliani, A.; Mackenzie, D. M. A.; Petersen, D. H.; Booth, T. J.; Bøggild, P. Graphene Transport Properties upon Exposure to PMMA Processing and Heat Treatments. 2D Mater. 2014, 1, 035005.

(31) van der Pauw, L. J. A Method of Measuring the Resistivity and Hall Coefficient on Lamellae of Arbitrary Shape. Philips Res. Rep. 1958, 13, 1-9.

(32) Mackenzie, D. M. A.; Buron, J. D.; Whelan, P. R.; Caridad, J. M.; Bjergfelt, M.; Luo, B.; Shivayogimath, A.; Smitshuysen, A. L.; Thomsen, J. D.; Booth, T. J.; et al. Quality Assessment of Graphene: Continuity, Uniformity, and Accuracy of Mobility Measurements. Nano Res. 2017, 10, 3596-3605.

(33) Schwierz, F. Graphene Transistors. Nat. Nanotechnol. 2010, 5, 487-496.

(34) Nguyen, V. L.; Shin, B. G.; Duong, D. L.; Kim, S. T.; Perello, D.; Lim, Y. J.; Yuan, Q. H.; Ding, F.; Jeong, H. Y.; Shin, H. S.; et al. Seamless Stitching of Graphene Domains on Polished Copper (111) Foil. Adv. Mater. 2015, 27, 1376-1382.

(35) Xu, X.; Zhang, Z.; Dong, J.; Yi, D.; Niu, J.; Wu, M.; Lin, L.; Yin, R.; Li, M.; Zhou, J.; et al. Ultrafast Epitaxial Growth of Metre-Sized Single-Crystal Graphene on Industrial Cu Foil. Sci. Bull. 2017, 62, 1074-1080.

(36) Jang, S. K.; Youn, J.; Song, Y. J.; Lee, S. Synthesis and Characterization of Hexagonal Boron Nitride as a Gate Dielectric. Sci. Rep. 2016, 6, 30449.

(37) Kim, S. M.; Hsu, A.; Park, M. H.; Chae, S. H.; Yun, S. J.; Lee, J. S.; Cho, D.-H.; Fang, W.; Lee, C.; Palacios, T.; et al. Synthesis of Large-Area Multilayer Hexagonal Boron Nitride for High Material Performance. Nat. Commun. 2015, 6, 8662.

(38) Kim, S.; Zhao, P.; Aikawa, S.; Einarsson, E.; Chiashi, S.; Maruyama, S. Highly Stable and Tunable N-Type Graphene FieldEffect Transistors with Poly(Vinyl Alcohol) Films. ACS Appl. Mater. Interfaces 2015, 7, 9702-9708.

(39) Liang, X.; Sperling, B. A.; Calizo, I.; Cheng, G.; Hacker, C. A.; Zhang, Q.; Obeng, Y.; Yan, K.; Peng, H.; Li, Q.; et al. Toward Clean and Crackless Transfer of Graphene. ACS Nano 2011, 5, 9144-9153.

(40) Isacsson, A.; Cummings, A. W.; Colombo, L.; Colombo, L.; Kinaret, J. M.; Roche, S. Scaling Properties of Polycrystalline Graphene: A Review. 2D Mater. 2016, 4, 012002.

(41) Vinogradov, N. A.; Zakharov, A. A.; Ng, M. L.; Mikkelsen, A.; Lundgren, E.; Mårtensson, N.; Preobrajenski, A. B. One-Dimensional Corrugation of the h -BN Monolayer on Fe(110). Langmuir 2012, 28, $1775-1781$. 